\title{
Sequence variation between the mouse and human glucose-6-phosphatase catalytic subunit gene promoters results in differential activation by peroxisome proliferator activated receptor gamma coactivator-1 $\alpha$
}

\author{
M. M. Schilling • J. K. Oeser • J. K. Chandy • \\ B. P. Flemming $\cdot$ S. R. Allen $\cdot$ R. M. O'Brien
}

Received: 17 January 2008 / Accepted: 18 April 2008 / Published online: 19 June 2008

(C) Springer-Verlag 2008

\begin{abstract}
Aims/hypothesis The glucose-6-phosphatase catalytic subunit (G6PC) plays a key role in hepatic glucose production by catalysing the final step in gluconeogenesis and glycogenolysis. Peroxisome proliferator activated receptor gamma coactivator- $1 \alpha$ (PGC-1 $\alpha)$ stimulates mouse G6pcluciferase fusion gene expression through hepatocyte nuclear factor- $4 \alpha$ (HNF-4 $\alpha$ ), which binds an element located between -76 and -64 in the promoter. The aim of this study was to compare the regulation of mouse $G 6 p c$ and human $G 6 P C$ gene expression by PGC- $1 \alpha$.

Methods PGC- $1 \alpha$ action was analysed by transient transfection and gel retardation assays.

Results In H4IIE cells, PGC- $1 \alpha$ alone failed to stimulate human $G 6 P C$-luciferase fusion gene expression even though the sequence of the -76 to $-64 \mathrm{HNF}-4 \alpha$ binding site is perfectly conserved in the human promoter. This difference could be explained, in part, by a 3 bp sequence variation between the mouse and human promoters. Introducing the human sequence into the mouse G6pc promoter reduced PGC- $1 \alpha$-stimulated fusion gene expression, whereas the inverse experiment, in which the mouse sequence was introduced into the human $G 6 P C$ promoter, resulted in the generation of a $G 6 P C$-luciferase fusion gene that was now induced by PGC- $1 \alpha$. This critical 3 bp region is located immediately adjacent to a consensus nuclear hormone receptor half-site that is perfectly conserved
\end{abstract}

M. M. Schilling $\cdot$ J. K. Oeser $\cdot$ J. K. Chandy $\cdot$ B. P. Flemming $\cdot$

S. R. Allen $\cdot$ R. M. O'Brien $(\varangle)$

Department of Molecular Physiology and Biophysics,

Vanderbilt University Medical School,

8415 MRB IV, 2213 Garland Ave,

Nashville, TN 37232-0615, USA

e-mail: richard.obrien@vanderbilt.edu between the mouse G6pc and human G6PC promoters. Gel retardation experiments revealed that this 3 bp region influences the affinity of HNF- $4 \alpha$ binding to the half-site. Conclusions/interpretation These observations suggest that PGC- $1 \alpha$ may be more important in the control of mouse $G 6 p c$ than human $G 6 P C$ gene expression.

Keywords Diabetes · Glucose-6-phosphatase · HNF-4alpha $\cdot$ PGC-1 alpha $\cdot$ Promoter $\cdot$ Transcription

\begin{tabular}{|c|c|}
\hline \multicolumn{2}{|c|}{ Abbreviations } \\
\hline FOXO1 & forkhead box $\mathrm{O} 1$ \\
\hline G6PC & glucose-6-phosphatase catalytic subunit \\
\hline HGP & hepatic glucose production \\
\hline $\mathrm{HNF}-4 \alpha$ & hepatocyte nuclear factor $-4 \alpha$ \\
\hline PGC- $1 \alpha$ & $\begin{array}{l}\text { peroxisome proliferator activated receptor } \\
\text { gamma coactivator- } 1 \alpha\end{array}$ \\
\hline PPAR & peroxisome proliferator activated receptor \\
\hline $5^{\prime}$ UTR & $5^{\prime}$ untranslated region \\
\hline
\end{tabular}

\section{Introduction}

Glucose-6-phosphatase catalyses the terminal step of gluconeogenesis and glycogenolysis, the hydrolysis of inorganic phosphate from glucose-6-phosphate [1]. Glucose-6-phosphatase is a multi-component enzyme system consisting of transporters for glucose-6-phosphate, glucose and inorganic phosphate, and a catalytic subunit whose active site is orientated towards the lumen of the endoplasmic reticulum [1]. In mammals, glucose-6-phosphatase activity is highest in the liver and kidney, but activity is also detected at lower levels in the brain, small intestine and 
pancreatic islets [1]. Hepatic glucose-6-phosphatase activity is critical for maintaining plasma glucose levels between meals. Mutations in the gene encoding the glucose-6phosphatase catalytic subunit (G6PC) result in glycogen storage disease type 1a (von Gierke's disease), which is characterised by life-threatening hypoglycaemia, growth retardation, renal dysfunction and hepatomegaly [2]. In contrast, increased G6PC mRNA and G6PC activity are thought to contribute to the elevated hepatic glucose production (HGP) characteristic of type 1 and type 2 diabetes [3, 4].

Peroxisome proliferator activated receptor (PPAR)-gammacoactivator $1 \alpha(\mathrm{PGC}-1 \alpha)$ is an important regulator of metabolism, contributing to transcriptional activation of genes involved in adaptive thermogenesis, mitochondrial biogenesis, respiration and gluconeogenesis $[5,6]$. In the liver PGC- $1 \alpha$ is upregulated during fasting and in diabetes and it stimulates expression of the G6PC and PEPCK (also known as $P C K 1)$ genes [7]. Two groups of investigators have generated mice with a global deletion of the $P g c-1 \alpha$ gene. Both reported that the mice were viable, but had multisystem abnormalities that included diminished mitochondrial respiratory capacity, vacuolar lesions in the central nervous system and cold intolerance $[8,9]$. However, these groups reported variable effects of PGC- $1 \alpha$ deletion on hepatic gluconeogenic gene expression and fasting blood glucose levels. Lin et al. [8] observed hypoglycaemia in the fasted state and reduced HGP. However, instead of the expected impairment, G6pc and Pepck gene expression were surprisingly elevated in the fed state to a level equivalent to that in the fasted state. This elevation of G6pc and Pepck expression was potentially caused by elevated hepatic $C / e b p \beta$ (also known as $C e b p b$ ) gene expression [8]. In contrast, in their mice, Leone et al. [9] observed euglycaemia in the fasted state with no elevation of $G 6 p c$, Pepck or $C / e b p \beta$ expression in the fed state and the same induction following fasting as seen in wild-type mice. The reasons for these differences are unclear. Interestingly, although Leone et al. [9] observed no change in G6pc and Pepck expression and euglycaemia in the fasted state, HGP and gluconeogenic flux are altered in these mice as a consequence of altered tricarboxylic acid cycle flux [10]. In mice with a liver-specific deletion of the $P g c-1 \alpha$ gene, G6pc and Pepck expression were normal in the fed state but their induction by fasting was impaired [11]. The observation that the effect of fasting was not abolished is probably not explained by the presence of PGC- $1 \beta$ because, although induced by fasting [12], it only weakly activates G6pc and Pepck expression [13]. However, the observation is consistent with the fact that ongoing protein synthesis is not required for the hormonal regulation of these genes [14]. As such, PGC-1 $\alpha$ is not essential for G6pc and Pepck expression, but does play an important role as a transcriptional amplifier [15].
At the molecular level, PGC- $1 \alpha$ interacts with multiple transcription factors including $\operatorname{PPAR} \alpha, \operatorname{PPAR} \gamma$, nuclear respiratory factor-1, the glucocorticoid receptor, oestrogenrelated receptors, liver $\mathrm{X}$ receptor, pregnane $\mathrm{X}$ receptor, myocyte enhancer factor- 2 and hepatocyte nuclear factor$4 \alpha(\mathrm{HNF}-4 \alpha)[5,6] . \mathrm{HNF}-4 \alpha$ is a member of the steroid hormone receptor superfamily and is present in the liver, intestine, kidney, pancreatic islets, stomach and skin [16, 17]. $H n f-4 \alpha$ (also known as $H n f 4 a$ ) knockout mice have an embryonic lethal phenotype [18], whereas liver-specific $H n f-4 \alpha$-deficient mice exhibit defective lipid homeostasis and weight loss [19]. Mutation of HNF4A results in maturity onset diabetes of the young type 1 , which is also characterised by altered lipid homeostasis as well as defective glucose-stimulated insulin secretion [17]. HNF$4 \alpha$ is essential for the induction of G6pc and Pepck expression by PGC- $1 \alpha[20]$.

We have previously shown that in rat H4IIE hepatoma cells the activation of mouse $G 6 p c$ expression by PGC- $1 \alpha$ was mediated through an HNF- $4 \alpha$ binding site located between -76 and -64 [21], whereas Rhee et al. [20] showed in immortalised mouse hepatocytes that the activation of human G6PC expression by PGC- $1 \alpha$ was mainly mediated through two HNF- $4 \alpha$ binding sites located between -296 and -284 , and -256 and -244 . Because these latter sites are poorly conserved in the mouse G6pc promoter, this observation appeared to explain the differences between the reported results. However, in the current study we demonstrate that the PGC- $1 \alpha$ responsiveness of the mouse $G 6 p c$ and human $G 6 P C$ promoters are fundamentally different. Thus, when the mouse $G 6 p c$ and human $G 6 P C$ promoters were compared in the same H4IIE cell line only mouse $G 6 p c-$ luciferase fusion gene expression was induced by PGC- $1 \alpha$. The molecular basis for this difference was mapped to a sequence variation between the mouse and human promoters that affects the affinity of HNF- $4 \alpha$ binding. These results suggest that PGC- $1 \alpha$ may be more important for the regulation of mouse $G 6 p c$ than human $G 6 P C$ expression, a difference that could contribute to the observed higher rate of fasting HGP in mice than humans.

\section{Methods}

Plasmid construction The generation of a mouse G6pcluciferase fusion gene, containing promoter sequence located between -484 and +66 , relative to the transcription start site, in the pGL3 MOD vector has been previously described [22]. PCR reactions with this plasmid as the template were used to generate mouse $G 6 p c$-luciferase fusion genes containing wild-type or mutated promoter 
sequence between -85 and +1 . A human $G 6 P C-$ luciferase fusion gene, containing promoter sequence located between -826 and +63 , was generated by PCR using human genomic DNA as the template. PCR reactions with this plasmid as the template were used to generate human $G 6 P C$-luciferase fusion genes containing wild-type or mutated promoter sequence between -83 and +7 . All promoter fragments generated by PCR were completely sequenced to verify the absence of polymerase errors.

Expression vectors encoding the rat glucocorticoid receptor (pRSV-GR) [23], rat HNF-4 $\alpha$ (pSG5-HNF4 $\alpha$ ) [24]) and human forkhead box O1 (FOXO1; pcDNA3FKHR) [25] were generously provided by K. Yamamoto (University of California, San Francisco, CA, USA), D. Granner (Vanderbilt University School of Medicine, Nashville, TN, USA) and D. Powell (Baylor College of Medicine, Houston, TX, USA), respectively.

A pBJ5 expression vector encoding a putative mouse PGC- $1 \alpha$ splice variant representing the entire 797 amino acid PGC- $1 \alpha$ open reading frame [26] minus amino acids 476 to 487 has been previously described [21]. A fulllength pBJ5-PGC-1 $\alpha$ expression vector was constructed by inserting the missing PGC- $1 \alpha$ sequence, which was isolated from I.M.A.G.E. clone \# 4193174 (Open Biosystems, Huntsville, AL, USA).

Cell culture and transient transfections Rat H4IIE hepatoma cells were cultured and transiently transfected in suspension using the calcium phosphate-DNA co-precipitation method as previously described [27].

Luciferase assays Luciferase assays were performed using the Dual-Luciferase Reporter Assay System (Promega Corp., Madison, WI, USA) according to the manufacturer's instructions. In these experiments we saw no effect of PGC$1 \alpha$ or HNF- $4 \alpha$ overexpression on Renilla luciferase activity. Therefore, for comparisons of PGC- $1 \alpha$ - and $\mathrm{HNF}-4 \alpha$-regulated gene expression, firefly luciferase activity directed by the various fusion gene constructs was expressed relative to Renilla luciferase activity in the same sample. Each construct was analysed in duplicate in multiple transfections, as specified in the figure legends, using multiple independent plasmid preparations.

Nuclear extract preparation and gel retardation assays H4IIE nuclear extract was prepared as previously described [28]. Oligonucleotides representing sense and antisense wildtype and mutated $G 6 p c$ promoter sequences as described in the text were synthesised with BamHI compatible ends by Operon Technologies (Alameda, CA, USA) and subsequently annealed and labelled with $\left[\alpha^{32} \mathrm{P}\right] \mathrm{dATP}$ using the Klenow fragment of Escherichia coli DNA Polymerase I to a specific activity of approximately $9 \times 10^{4} \mathrm{~Bq} / \mathrm{pmol}$ [29].
Gel retardation assays were then performed exactly as previously described [21].

Statistical analyses The transfection data were analysed for differences from the control values, as specified in the figure legends. Statistical comparisons were calculated using an unpaired Student's $t$ test. The level of significance was as indicated (two-sided test).

\section{Results}

$P G C-1 \alpha$ can selectively stimulate mouse G6pc-luciferase fusion gene expression The rate of fasting HGP is tenfold higher in mice than humans $[30,31]$. We have been interested as to whether differences in the regulation of G6PC gene transcription between mice and humans could partly explain this observation. A carbohydrate response element identified in the mouse and rat $G 6 p c$ promoters is not conserved in the human G6PC promoter [32]. However, the mechanism by which insulin suppresses both mouse $G 6 p c$ - and human $G 6 P C$-luciferase fusion gene expression in human HepG2 hepatoma cells appears identical [33]. Likewise, the synthetic glucocorticoid, dexamethasone, equally stimulates mouse $G 6 p c-$ and human $G 6 P C-$ luciferase fusion gene expression in rat H4IIE hepatoma cells (Fig. 1). Since PGC-1 $\alpha$ markedly induces G6PC expression and HGP [7], we next compared the ability of PGC- $1 \alpha$ to stimulate mouse $G 6 p c-$ and human $G 6 P C-$ luciferase fusion gene expression in rat H4IIE hepatoma cells. As previously reported [21], co-transfection of H4IIE cells with an expression vector encoding PGC- $1 \alpha$ robustly induces the expression of a mouse $G 6 p c$-luciferase fusion gene containing promoter sequence between -484 and +66 (Fig. 2a). In contrast, expression of a human G6PC -luciferase fusion gene containing promoter sequence between -826 and +63 was unaffected (Fig. 2a). Although mouse PGC- $1 \alpha$ was used in this experiment, mouse and human PGC- $1 \alpha$ share $95 \%$ amino acid sequence homology (758/797), suggesting that limited differences in structural characteristics and binding properties exist between the two species. Indeed, the observation that the human promoter was unresponsive to PGC- $1 \alpha$ at all concentrations tested (Fig. 2b) suggested that there is a fundamental difference between the mouse G6pc and human G6PC promoters rather than just a difference in sensitivity towards the action of PGC- $1 \alpha$.

Identification of sequence variations that contribute to the selective effect of $P G C-1 \alpha$ on mouse G6pc-luciferase fusion gene expression The results suggested that either the human promoter contained a species-specific element 


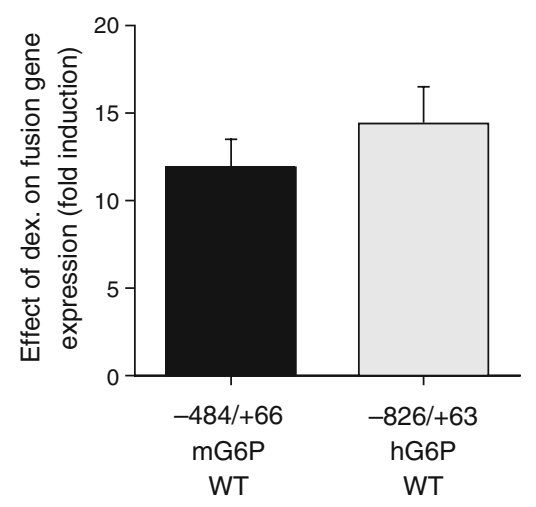

Fig. 1 Dexamethasone stimulates mouse G6pc- and human G6PCluciferase fusion gene expression. H4IIE cells were transiently cotransfected, as described in the Methods section, with the indicated mouse $G 6 p c$ - or human $G 6 P C$-luciferase fusion genes $(15 \mu \mathrm{g})$ and expression vectors encoding Renilla luciferase $(0.15 \mu \mathrm{g})$ and the glucocorticoid receptor $(5 \mu \mathrm{g})$. Following transfection, cells were incubated for 18 to $20 \mathrm{~h}$ in serum-free medium in the presence or absence of $500 \mathrm{nmol} / 1$ dexamethasone (dex.). Cells were then collected and luciferase activity assayed as described in the Methods section. Results are presented as the ratio of firefly luciferase activity, corrected for Renilla luciferase in the cell lysate, in dexamethasone vs control cells and are expressed as fold induction. Results represent the mean \pm SEM of three to eight experiments using three independent preparations of both fusion gene constructs in which each experimental condition was assayed in duplicate. hG6P, human G6PC; mG6P, mouse G6pc; WT, wild-type

that repressed the PGC- $1 \alpha$ response or that the mouse promoter contained a species-specific PGC- $1 \alpha$ responsive element that is absent in the human promoter. Before addressing these possibilities, we first sought to define shorter regions of the mouse $G 6 p c$ and human $G 6 P C$ promoters that demonstrated a selective effect of PGC- $1 \alpha$.

The effect of PGC- $1 \alpha$ on mouse G6pc-luciferase fusion gene expression was previously mapped to the promoter region between -85 and +66 and was shown to require an HNF- $4 \alpha$ binding site located between -76 and -64 [21]. The $5^{\prime}$ untranslated regions (5' UTRs) of the mouse and human genes contain numerous sequence variations, whereas the mouse promoter sequence between -85 and +1 and the equivalent human sequence between -83 and +1 are highly conserved (Fig. 3). Deletion of the mouse $5^{\prime}$ UTR between +2 and +66 reduced the magnitude of PGC$1 \alpha$-induced mouse $G 6 p c$-luciferase fusion gene expression indicating that element(s) in the $5^{\prime}$ UTR contribute to the PGC $-1 \alpha$ response (data not shown). However, the -85 to +1 promoter region still mediated a PGC- $1 \alpha$ response (Fig. 4a). In contrast, a similar region of the human G6PC promoter, located between -83 and +7 , was unresponsive to PGC- $1 \alpha$ (Fig. $4 \mathrm{~b}$ ). The $5^{\prime}$ UTR sequence between +1 and +7 is identical between mouse and human so this cannot explain the observed difference. This result implies that at least part of the differential regulation of mouse $G 6 p c-$ and human $G 6 P C$-luciferase fusion genes by PGC-1 $\alpha$ must be explained by sequence differences within the proximal promoter regions shown in Fig. 3.

Inspection of the mouse promoter sequence between -85 and +1 with the equivalent human sequence between -83 and +1 revealed only seven nucleotide differences, specifically one deletion and six substitutions (Fig. 3). The previously identified mouse HNF- $4 \alpha$ binding site [21] is

a

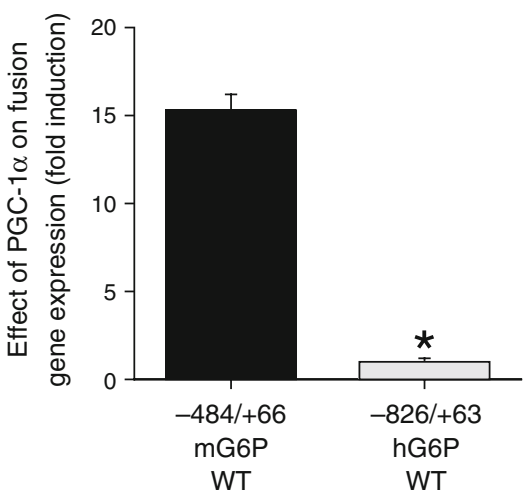

b

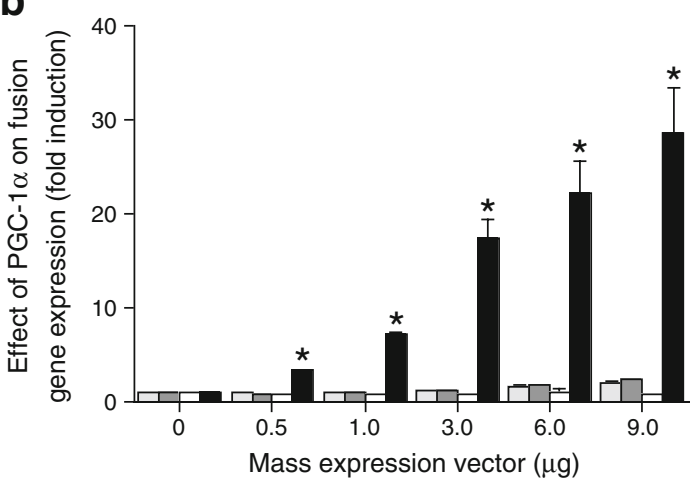

Fig. 2 PGC- $1 \alpha$ selectively stimulates mouse $G 6 p c-$ but not human $G 6 P C$-luciferase fusion gene expression. H4IIE cells were transiently co-transfected as described in the Methods section with the indicated mouse $G 6 p c$ - or human $G 6 P C$-luciferase fusion genes $(12 \mu \mathrm{g})$, an expression vector encoding Renilla luciferase $(0.15 \mu \mathrm{g})$ and either a pBJ5 expression vector encoding PGC- $1 \alpha$ or the empty pBJ5 vector control $(3 \mu \mathrm{g}[\mathbf{a}])$ or as indicated [b]). Following transfection, cells were incubated for 18 to $20 \mathrm{~h}$ in serum-free medium. Cells were then collected and luciferase activity assayed as described in the Methods section. Results are presented as the ratio of firefly luciferase activity, corrected for Renilla luciferase in the cell lysate, in PGC- $1 \alpha$ transfected vs empty vector-transfected cells and are expressed as fold induction. Results represent the mean \pm SEM of 12 to 35 experiments (a) or three experiments (b) using three independent preparations of both fusion gene constructs in which each experimental condition was assayed in duplicate. Statistical significance (a) $* p<$ 0.05 vs $-484 /+66$ mG6P WT; (b) $* p<0.05$ vs matching pBJ5. (b) white columns, $-484 /+66$ mG6P + pBJ5; black columns, $-484 /+66$ mG6P + pBJ5-PGC-1 $\alpha$; light grey columns, $-826 /+63$ hG6P + pBJ5; dark grey columns, $-826 /+63$ hG6P + pBJ5-PGC-1 $\alpha$. hG6P, human G6PC; mG6P, mouse G6pc; WT, wild-type 


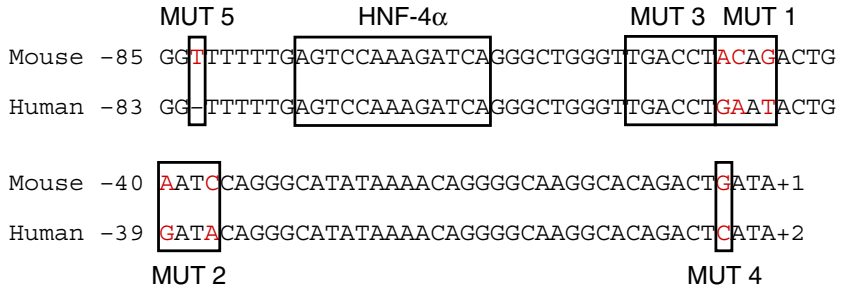

Fig. 3 Sequence alignment of the mouse $G 6 p c$ and human $G 6 P C$ gene promoters. The human $G 6 P C$ and mouse $G 6 p c$ proximal promoter sequences are labelled relative to the experimentally determined transcription start sites, designated as +1 [46, 47]. The conserved HNF- $4 \alpha$ binding site [21] is boxed. The G6PC promoter regions shown contain $7 \mathrm{bp}$ that differ between the human and mouse species. Site-directed mutations were generated in the mouse $G 6 p c$ and human $G 6 P C$ promoters, designated MUT 1, 2 and 4, in which the mouse sequence was substituted for that of the human and vice versa. In addition, a site-directed mutation of a conserved consensus nuclear receptor half-site, designated MUT 3, was generated in the mouse $G 6 p c$ promoter, in which the sequence of this half-site was changed to GTCAAG

perfectly conserved in the human promoter (Fig. 3). To identify the sequence required for the selective effect of PGC- $1 \alpha$ on mouse $G 6 p c-$ luciferase fusion gene expression site, directed mutations were generated in the context of the mouse -85 to +1 G6pc promoter, designated hMUT 1, 2 and 4 , in which the mouse sequence was substituted for that of the human (Fig. 3). G6pc-luciferase fusion genes containing these mutations were then transiently transfected into H4IIE cells and the effect of PGC- $1 \alpha$ co-transfection was assessed. Figure $4 \mathrm{a}$ shows that switching the sequence of the MUT 2 and MUT 4 regions to that of the human had no effect on the PGC- $1 \alpha$ response, whereas switching the sequence of the MUT 1 region to that of the human reduced the PGC- $1 \alpha$ response. Significantly, Fig. $4 b$ shows that when the inverse experiment was performed, in which the sequence of the MUT 1 region in the human promoter was switched to that of the mouse, expression of the resulting G6PC-luciferase fusion gene, designated $-83 /+7$ hG6P mMUT 1 , was induced by PGC- $1 \alpha$. This result suggests that the selective effect of PGC-1 $\alpha$ on mouse G6pc -luciferase fusion gene expression can be explained, at least in part, by the differential binding of a factor to the MUT 1 region.

A conserved nuclear receptor half-site is required for the effect of PGC-1 $\alpha$ on mouse G6pc-luciferase fusion gene expression Analysis of the G6pc promoter sequence encompassing the MUT 1 region using MatInspector software [34] revealed the existence of a consensus nuclear receptor half-site adjacent to the MUT 1 region (Fig. 3). This element is perfectly conserved in the mouse $G 6 p c$ and human $G 6 P C$ promoters (Fig. 3). A mouse $G 6 p c-$ luciferase fusion gene containing a site-directed mutation of this element, designated $-85 /+1$ mG6p MUT 3, was transiently transfected into H4IIE cells and the effect of PGC- $1 \alpha$ cotransfection was assessed. Figure 5 shows that mutating the MUT 3 region markedly reduced the PGC- $1 \alpha$ response. These data demonstrated that, in addition to the HNF- $4 \alpha$ binding site located between -76 and -64 [21], this nuclear receptor half-site was critical for the PGC- $1 \alpha$ response.

Altered HNF-4 $\alpha$ binding to a conserved nuclear receptor half-site contributes to the selective effect of $P G C-1 \alpha$ on mouse G6pc-luciferase fusion gene expression We reasoned that the scenario most likely to explain the data was one in which PGC- $1 \alpha$ interacted with a member of the nuclear receptor superfamily bound to the MUT 3 region with the flanking sequence, as represented by the MUT 1 region, affecting binding affinity. Studies on PPAR $\alpha$ binding to nuclear receptor half-sites have established a precedent for such a model [35]. Gel retardation assays using the mouse $G 6 p c$ promoter sequence between -62 and -29 , which includes both the MUT 3 and MUT 1 regions (Fig. 3), as the labelled probe failed to detect specific protein-DNA binding (data not shown). However, this presumably reflects the low affinity of interaction of nuclear receptors with half-sites.

Since chromatin immunoprecipitation experiments have shown that HNF- $4 \alpha$ binds this region of the G6pc promoter in intact H4IIE cells [21] the simplest model to explain the data is one in which HNF- $4 \alpha$ binds the $-76 /-64$ element as well as the MUT 3 region, with the MUT 1 flanking region affecting the affinity of HNF- $4 \alpha$ binding (Fig. 3). To explore this possibility an oligonucleotide representing the mouse $G 6 p c$ promoter sequence between -82 and -37 , which contains the high-affinity $-76 /-64 \mathrm{HNF}-4 \alpha$ binding site [21] and the putative HNF- $4 \alpha$ binding half-site (Fig. 6a), was used as the labelled probe in gel retardation assays. The aim was to assess the ability of oligonucleotides representing the mouse $G 6 p c$ promoter sequence between -82 and -55 , which includes just the high-affinity $-76 /-64$ HNF- $4 \alpha$ binding site, or the mouse $G 6 p c$ promoter sequence between -62 and -29 , which includes just the MUT 3 and MUT 1 regions (Fig. 6a), to compete for HNF- $4 \alpha$ binding. When the labelled double-stranded wild-type $G 6 P C-82$ and -37 oligonucleotide was incubated with nuclear extract prepared from H4IIE cells, a single major protein-DNA complex was detected (Fig. 6b). Supershift experiments using specific antisera have demonstrated that this complex contains HNF$4 \alpha$ [21]. Competition experiments, in which a 100 -fold molar excess of unlabelled DNA was included with the labelled probe, were used to correlate protein binding with the PGC- $1 \alpha$ response. The wild-type G6PC $-82 /-37$ oligonucleotide competed effectively for formation of the protein-DNA complex, as did the $-82 /-55$ oligonucleotide that includes just the high-affinity $-76 /-64 \mathrm{HNF}-4 \alpha$ binding site (Fig. 6b). Significantly, the wild-type G6PC $-62 /-29$ 
a

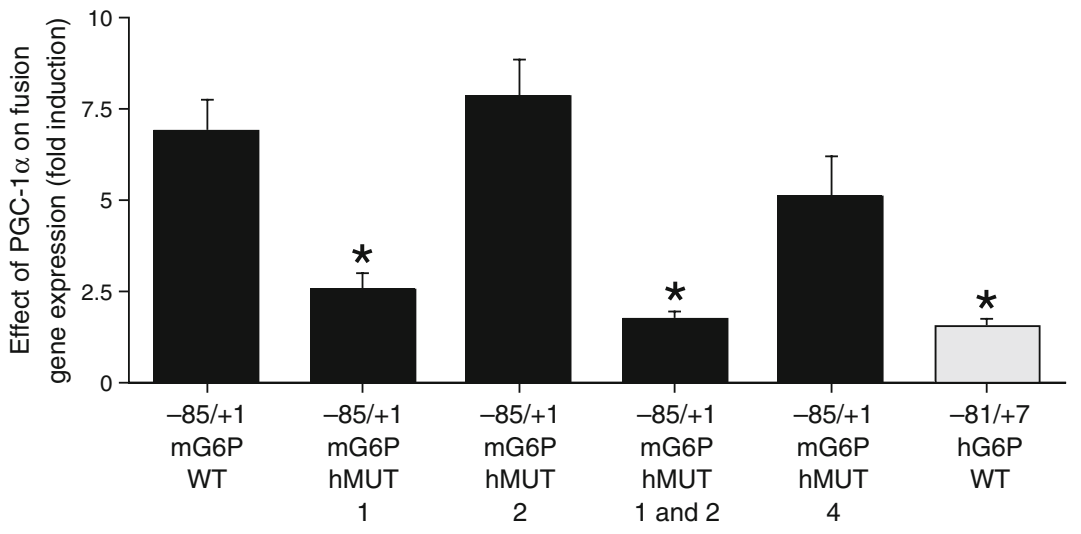

b

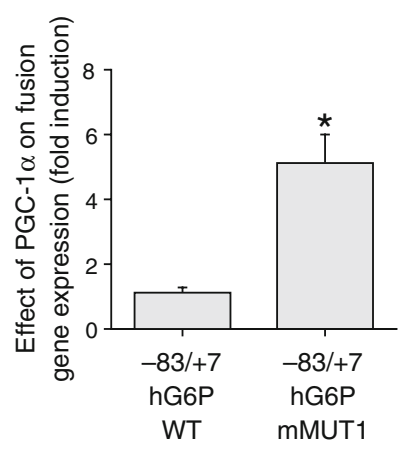

Fig. 4 Identification of sequences that contribute to the selective stimulation of mouse $G 6 p c-$ but not human $G 6 P C$-luciferase fusion gene expression by PGC- $1 \alpha$. H4IIE cells were transiently cotransfected, as described (Methods section) with the indicated mouse $G 6 p c-(\mathbf{a})$ or human (a, b) G6PC-luciferase fusion genes $(12 \mu \mathrm{g})$, an expression vector encoding Renilla luciferase $(0.15 \mu \mathrm{g})$ and either a pBJ5 expression vector encoding PGC- $1 \alpha$ or the empty pBJ5 vector control $(3 \mu \mathrm{g})$. The fusion genes contained either the indicated wildtype mouse and human promoter regions or site-directed mutations of these promoters as described in the text. These mutations represented substitutions of mouse sequence by that of the human (hMUT) or vice versa (mMUT) (Fig. 3). Following transfection, cells were incubated

oligonucleotide also competed for formation of the proteinDNA complex confirming that this promoter region can bind HNF-4 $\alpha$ (Fig. 6b). Oligonucleotides, designated mG6P hMUT 1 and MUT 3 (Fig. 6a) that contain mutations identical to those described in the hMUT 1 (Fig. 4a) and MUT 3 (Fig. 5) fusion genes, competed poorly with the labelled probe for protein binding and less effectively than the WT G6P $-62 /-29$ oligonucleotide (Fig. 6b). Figure 6c shows the results from multiple experiments using a variable molar excess of the various competitors. The observation that the MUT 3 oligonucleotide still competed slightly at high molar excess may be explained by the presence of a partial nuclear receptor half-site, AGGgCA, located between -35 and -30 (Fig. 6a). These results show that the binding of HNF- $4 \alpha$ to the MUT 1 and MUT 3 regions correlates with the PGC- $1 \alpha$ response.

In an attempt to further support this conclusion, protein binding to labelled probes representing the mouse $-82 /-37$ region and equivalent human $-81 /-36$ region was compared. No difference was detected (data not shown), suggesting that the use of labelled probes only detects HNF- $4 \alpha$ binding to the conserved high-affinity HNF- $4 \alpha$ binding site, and not the HNF- $4 \alpha$ half-site. This is consistent with the observation that protein binding to the labelled $-62 /-29$ probe was not detectable (see above). This result indicates that, while HNF- $4 \alpha$ binding to the half-site can be implied from competition experiments (Fig. 6), it cannot be directly demonstrated using labelled probes in the gel retardation

for 18 to $20 \mathrm{~h}$ in serum-free medium. Cells were then collected and luciferase activity assayed as described in the Methods section. Results are presented as the ratio of firefly luciferase activity, corrected for Renilla luciferase in the cell lysate, in PGC- $1 \alpha$ transfected vs empty vector-transfected cells and are expressed as fold induction. Results represent the mean \pm SEM of six to nine experiments (a) or three experiments (b) using three independent preparations of all fusion gene constructs in which each experimental condition was assayed in duplicate. Statistical significance (a) ${ }^{*} p<$ 0.05 vs $-85 /+1$ mG6P WT; (b) ${ }^{*} p<0.05$ vs $-83 /+7$ hG6P WT. hG6P, human $G 6 P C$; mG6P, mouse G6pc; WT, wild-type

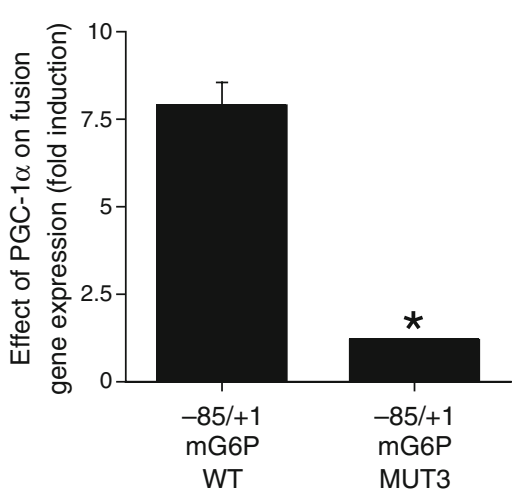

Fig. 5 A conserved nuclear receptor half-site is required for the stimulation of $G 6 p c-$ luciferase fusion gene expression by PGC- $1 \alpha$. H4IIE cells were transiently co-transfected as described in the Methods with the indicated mouse $G 6 p c$-luciferase fusion genes $(12 \mu \mathrm{g})$, an expression vector encoding Renilla luciferase $(0.15 \mu \mathrm{g})$ and either a pBJ5 expression vector encoding PGC- $1 \alpha$ or the empty pBJ5 vector control $(3 \mu \mathrm{g})$. The fusion genes contained either the wild-type mouse promoter sequence between -85 and +1 or a sitedirected mutation of the conserved nuclear receptor half-site (MUT 3), as described (Fig. 3). Following transfection, cells were incubated for 18 to $20 \mathrm{~h}$ in serum-free medium. Cells were then collected and luciferase activity assayed as described in the Methods. Results are presented as the ratio of firefly luciferase activity, corrected for Renilla luciferase in the cell lysate, in PGC-1 $\alpha$-transfected vs empty vectortransfected cells and are expressed as fold induction. Results represent the mean \pm SEM of seven experiments using three independent preparations of both fusion gene constructs in which each experimental condition was assayed in duplicate. ${ }^{*} p<0.05$ vs $-85 /+1$ mG6P WT. mG6P, mouse G6pc; WT, wild-type 
a

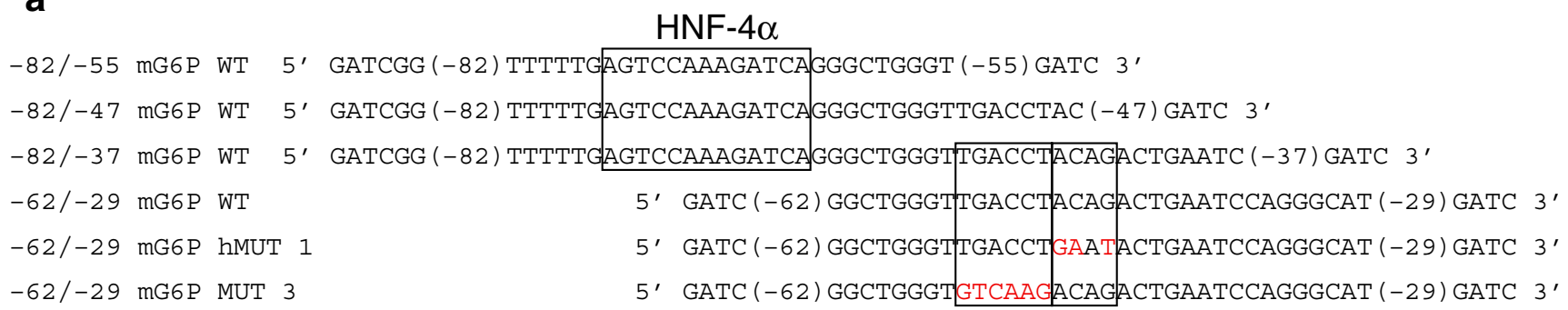

\section{MUT 3 MUT 1}

b

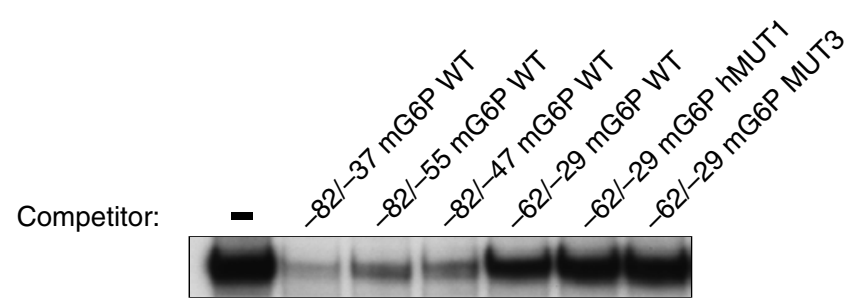

C

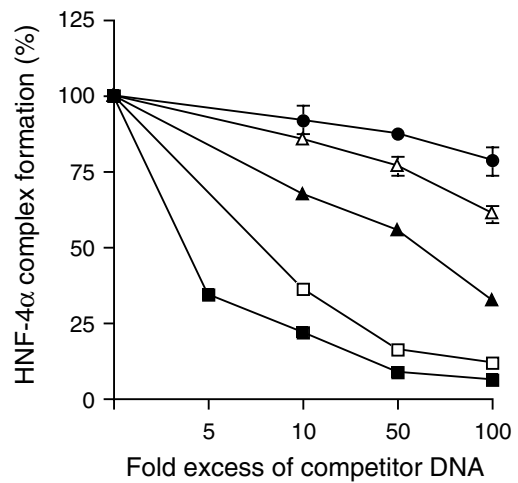

protein binding analysed using the gel retardation assay as described in the Methods. In the representative autoradiograph shown only the retarded complex is visible and not the free probe, which was present in excess. Previous experiments have shown that the specific complex detected represents HNF- $4 \alpha$ binding [21]. c Gel retardation experiments were performed as described (b) except that a variable molar excess of unlabelled competitor DNAs was used as shown and data were quantified using scintillation counting. Results represent the mean \pm SEM of at least three experiments. Black squares, $-82 /-37$ mG6P WT; white squares, $-82 /-55$ mG6P WT; black triangles, $-62 /-29$ mG6P WT; white triangles, $-62 /-29 \mathrm{mG} 6 \mathrm{P}$ hMUT1; circles, $-62 /-29$ mG6P MUT3. hMUT, human mutation; mG6P, mouse G6pc

case. Although PGC- $1 \alpha$ and HNF- $4 \alpha$ had little effect alone, co-transfection of both PGC- $1 \alpha$ and HNF- $4 \alpha$ together resulted in a synergistic induction of human G6PC-luciferase fusion gene expression (Fig. 7). The effects of both HNF- $4 \alpha$ and PGC- $1 \alpha$ alone or in combination were enhanced when the sequence of the MUT 1 region in the human promoter was switched to that of the mouse (Fig. 7). Similarly, co-transfection of PGC- $1 \alpha$ and HNF- $4 \alpha$ also resulted in a synergistic induction of mouse $G 6 p c$-luciferase fusion gene expression, but in this case the effects of HNF- $4 \alpha$ and PGC- $1 \alpha$, either alone or in combination, were reduced when the sequence of the MUT 1 region in the mouse promoter was switched to that of the human (Fig. 7). This again suggests that the sequence of the MUT 1 region affects both the affinity of HNF- $4 \alpha$ binding and the PGC- $1 \alpha$ response. The observation that overexpression of HNF- $4 \alpha$ affects the PGC- $1 \alpha$ response implies that the results of $G 6 P C$-luciferase fusion gene flanking sequence. Figure 7 shows that this is indeed the 


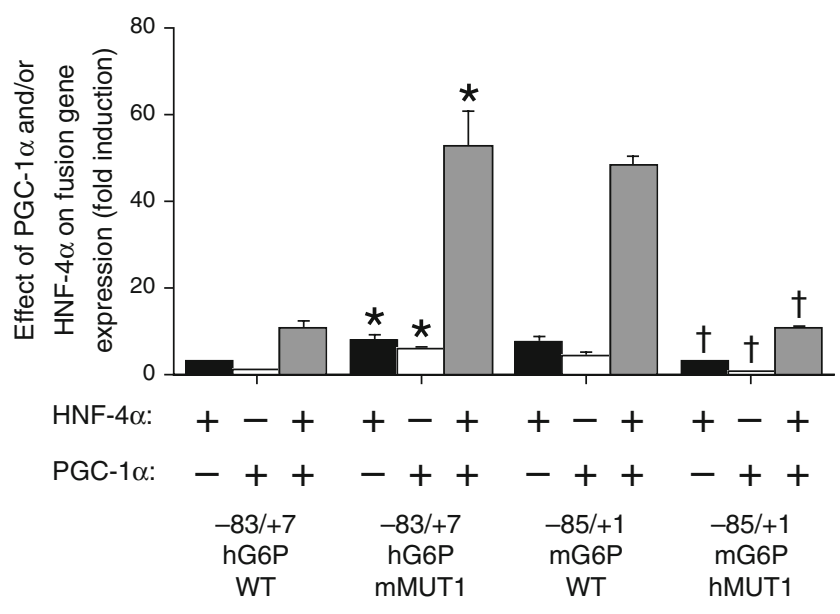

Fig. 7 Combined expression of $\mathrm{HNF}-4 \alpha$ and PGC- $1 \alpha$ synergistically stimulates both mouse $G 6 p c-$ and human $G 6 P C$-luciferase fusion gene expression. H4IIE cells were transiently co-transfected as described in the Methods with the indicated mouse $G 6 p c-$ or human $G 6 P C$-luciferase fusion genes $(12 \mu \mathrm{g})$ and expression vectors encoding Renilla luciferase $(0.15 \mu \mathrm{g})$ and either pBJ5 $(3 \mu \mathrm{g})$ or pBJ5-PGC-1 $\alpha(3 \mu \mathrm{g})$, or pSG5 $(3 \mu \mathrm{g})$ or pSG5-HNF-4 $\alpha(3 \mu \mathrm{g})$. Following transfection the cells were incubated for 18 to $20 \mathrm{~h}$ in serum-free medium. The cells were then collected and luciferase assays were performed as described in the Methods. Results are presented as the ratio of firefly luciferase activity, corrected for Renilla luciferase activity in the cell lysate, in HNF- $4 \alpha$ - and/or PGC- $1 \alpha$ stimulated vs empty vector-transfected cells, expressed as fold induction. Results represent the mean $\pm \mathrm{SEM}$ of three experiments using three independent preparations of the fusion gene plasmids with each sample assayed in duplicate. ${ }^{*} p<0.05$ vs $-83 /+7 \mathrm{hG6P} \mathrm{WT;}{ }^{\dagger} p<0.05$ vs $-85 /+1$ mG6P WT. Black columns, pSG5-HNF-4 $\alpha$; white columns, pBJ5-PGC-1 $\alpha$; grey columns, pSG5-HNF- $4 \alpha+$ pBJ5-PGC-1 $\alpha$. hG6P, human $G 6 P C$; hMUT, human mutation; mG6P, mouse G6pc; mMUT, mouse mutation; WT, wild-type

analyses will be dependent on variations in endogenous HNF- $4 \alpha$ levels in H4IIE cells. This may explain why in some experiments the presence of the human MUT 1 sequence is associated with a complete lack of a PGC- $1 \alpha$ response (Fig. 4b) whereas in other experiments a partial response remains (Fig. 4a).

Figure 8 shows similar experiments, in which the action of PGC- $1 \alpha$ on $G 6 P C$-luciferase fusion gene expression was assessed in the presence or absence of FOXO1. As previously reported, both mouse $G 6 p c-[38]$ and human [39] $G 6 P C$-luciferase fusion gene expression was stimulated by overexpression of FOXO1 (Fig. 8). In addition, cotransfection of PGC- $1 \alpha$ and FOXO1 together resulted in a synergistic induction of mouse $G 6 p c-$ and human $G 6 P C-$ luciferase fusion gene expression (Fig. 8).

\section{Discussion}

Our results demonstrate that, in H4IIE cells, PGC- $1 \alpha$ can selectively stimulate mouse $G 6 p c-$ but not human $G 6 P C-$ luciferase fusion gene expression (Fig. 2). This differential regulation can be explained by a sequence variation between the mouse and human promoters that results in reduced binding of HNF- $4 \alpha$ to the human promoter (Figs 3, 4, 5 and 6). When HNF- $4 \alpha$ (Fig. 7) or FOXO1 (Fig. 8) are overexpressed, PGC- $1 \alpha$ interacts synergistically with these factors to induce human $G 6 P C$-luciferase fusion gene expression, although the induction achieved is still less pronounced than that seen with the mouse promoter. These results imply that PGC- $1 \alpha$ may be more important for the regulation of $G 6 P C$ expression in mice than in humans and that the difference in PGC- $1 \alpha$ regulation reported here has the potential to contribute to the tenfold higher rate of HGP in mice $[30,31]$. In addition, the results suggest that the regulation of $G 6 P C$ expression by PGC- $1 \alpha$ is likely to be more sensitive to changes in levels of HNF- $4 \alpha$ and FOXO1 in humans than mice.

Spiegelman and colleagues [20, 40] previously noted that PGC- $1 \alpha$ alone had little effect on human $G 6 P C$ luciferase fusion gene expression in immortalised mouse hepatocytes and the data presented here provide an

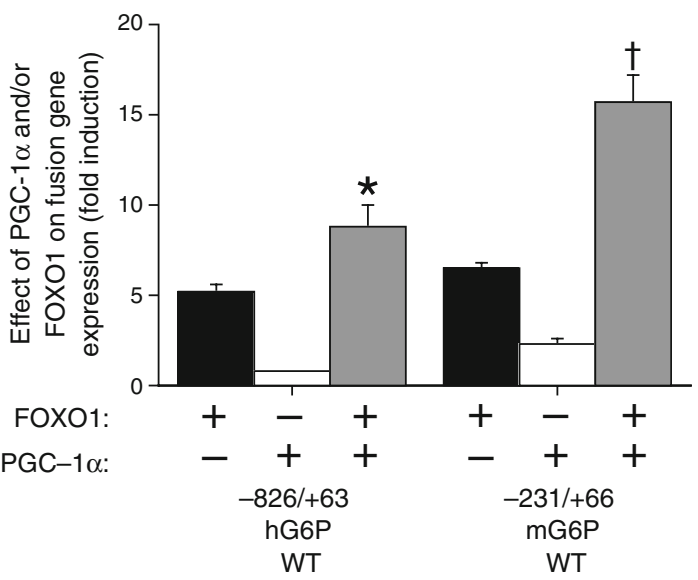

Fig. 8 Combined expression of FOXO1 and PGC- $1 \alpha$ synergistically stimulates both mouse $G 6 p c-$ and human $G 6 P C$-luciferase fusion gene expression. H4IIE cells were transiently co-transfected as described in the Methods section with the indicated mouse $G 6 p c-$ or human $G 6 P C$-luciferase fusion genes $(12 \mu \mathrm{g})$ and expression vectors encoding Renilla luciferase $(0.15 \mu \mathrm{g})$ and either pBJ5 $(1.5 \mu \mathrm{g})$ or pBJ5-PGC-1 $\alpha(1.5 \mu \mathrm{g})$, or pcDNA3 $(1.5 \mu \mathrm{g})$ or pcDNA3-FOXO1 $(1.5 \mu \mathrm{g})$. Following transfection the cells were incubated for 18 to 20 $\mathrm{h}$ in serum-free medium. The cells were then collected and luciferase assays were performed as described in the Methods. Results are presented as the ratio of firefly luciferase activity, corrected for Renilla luciferase activity in the cell lysate, in FOXO1- and/or PGC-1 $\alpha$ stimulated vs empty vector-transfected cells, expressed as fold induction. Results represent the mean \pm SEM of nine experiments using three independent preparations of the fusion gene plasmids with each sample assayed in duplicate. ${ }^{*} p<0.05$ vs $-826 /+63$ hG6P WT + pcDNA3-FOXO1; ${ }^{\dagger} p<0.05$ vs $-231 /+66$ mG6P WT + pcDNA3FOXO1. Black columns, pcDNA-FOXO1; white columns, pBJ5PGC- $1 \alpha$; grey columns: pcDNA-FOXO1 + pBJ5-PGC- $1 \alpha$. hG6P, human G6PC; mG6P, mouse G6PC; WT, wild-type 
explanation for that observation. They also reported that cotransfection of PGC-1 $1 \alpha$ with either FOXO1 [40] or HNF$4 \alpha[20]$ resulted in a synergistic induction of fusion gene expression. The same synergistic interaction between PGC$1 \alpha$ and HNF- $4 \alpha$ (Fig. 7) and FOXO1 (Fig. 8) is seen in H4IIE cells. With respect to the action of FOXO1, Puigserver et al. [40] presented a model in which PGC-1 $\alpha$ interacted directly with FOXO1. However, mutation [20] or deletion [21] of the three insulin response sequences in the G6PC promoter had no effect on the PGC- $1 \alpha$ response. Moreover, we recently showed that PGC- $1 \alpha$ stimulates $G 6 p c$-luciferase fusion gene expression through HNF- $4 \alpha$ and that the binding of FOXO1 to the G6pc promoter is neither required nor sufficient for this induction [41]. We hypothesise that the synergism seen between FOXO1 and PGC- $1 \alpha$ occurs at the level of the pre-initiation transcription complex. We envisage that both FOXO1, bound to multiple sites in the G6PC promoter, and PGC- $1 \alpha$ tethered to the promoter through HNF- $4 \alpha$ synergise to increase preinitiation complex formation. This concept is consistent with the observation that both proteins can interact with other non-DNA bound factors [5, 6, 42]. For example, FOXO1 can bind the CREB binding protein KIX domain, located within the N-terminal activation domain [43], whereas PGC- $1 \alpha$ can bind the C-terminal activation domain on the related protein p300 [44]. The synergistic action of FOXO1 and PGC- $1 \alpha$ on G6PC-luciferase fusion gene transcription could then feasibly be mediated through combined binding to CREB binding protein/p300. More recently, Matsumoto and colleagues [45] demonstrated in primary hepatocytes lacking FOXO1 that the induction of $G 6 P C$ expression by PGC- $1 \alpha$ was markedly reduced. This is consistent with the synergistic interaction between FOXO1 and PGC-1 $\alpha$ seen in both H4IIE cells [41] and immortalised mouse hepatocytes [40]. However, while the effect of PGC- $1 \alpha$ on $G 6 p c$ expression was markedly reduced in primary hepatocytes lacking FOXO1, a $\sim 20$ fold induction remained [45]. In contrast, the ability of PGC- $1 \alpha$ to stimulate $G 6 p c$ expression in hepatocytes derived from mice lacking HNF- $4 \alpha$ is completely lost [20]. The results of Matsumoto and colleagues [45] are therefore consistent with our model, in which PGC- $1 \alpha$ stimulates $G 6 P C$-luciferase fusion gene expression through HNF- $4 \alpha$, with the binding of FOXO1 to the G6PC promoter being neither required nor sufficient for this induction, although it does act synergistically to enhance the response [41].

Published reports suggest that this model is also consistent with the mechanism of action of PGC- $1 \alpha$ on $P E P C K$ expression. Thus, the action of PGC $-1 \alpha$ on $P E P C K$ fusion gene expression is mediated through HNF$4 \alpha$ and glucocorticoid receptor binding sites, whereas the FOXO1 binding site is not required for the PGC- $1 \alpha$ response $[7,15]$. In addition, the ability of PGC- $1 \alpha$ to stimulate Pepck expression in hepatocytes derived from mice lacking HNF- $4 \alpha$ is also completely lost [20].

Acknowledgements We thank J. N. Boustead for constructing the full-length PGC- $1 \alpha$ expression vector and several human $G 6 P C-$ luciferase fusion genes. We also thank K. Yamamoto, D. Granner and D. Powell for providing the glucocorticoid receptor, HNF- $4 \alpha$ and FOXO1 expression vectors, respectively. Research in the laboratory of R. M. O'Brien was supported by NIH grant DK56374 and by NIH grant P60 DK20593, which supports the Vanderbilt Diabetes Center Core Laboratory. M. M. Schilling was supported by the Vanderbilt Molecular Endocrinology Training Program (5 T 32 DK07563).

Duality of interest The authors declare that there is no duality of interest associated with this manuscript.

\section{References}

1. Van Schaftingen E, Gerin I (2002) The glucose-6-phosphatase system. Biochem J 362:513-532

2. Chou JY, Matern D, Mansfield BC, Chen YT (2002) Type I glycogen storage diseases: disorders of the glucose-6-phosphatase complex. Curr Mol Med 2:121-143

3. Barzilai N, Rossetti L (1993) Role of glucokinase and glucose-6phosphatase in the acute and chronic regulation of hepatic glucose fluxes by insulin. J Biol Chem 268:25019-25025

4. Cline GW, Rothman DL, Magnusson I, Katz LD, Shulman GI (1994) 13C-nuclear magnetic resonance spectroscopy studies of hepatic glucose metabolism in normal subjects and subjects with insulin-dependent diabetes mellitus. J Clin Invest 94:2369-2376

5. Lin J, Handschin C, Spiegelman BM (2005) Metabolic control through the PGC-1 family of transcription coactivators. Cell Metab 1:361-370

6. Finck BN, Kelly DP (2006) PGC-1 coactivators: inducible regulators of energy metabolism in health and disease. J Clin Invest 116:615-622

7. Yoon JC, Puigserver P, Chen G et al (2001) Control of hepatic gluconeogenesis through the transcriptional coactivator PGC-1. Nature 413:131-138

8. Lin J, Wu PH, Tarr PT et al (2004) Defects in adaptive energy metabolism with CNS-linked hyperactivity in PGC-1alpha null mice. Cell 119:121-135

9. Leone TC, Lehman JJ, Finck BN et al (2005) PGC-1alpha deficiency causes multi-system energy metabolic derangements: muscle dysfunction, abnormal weight control and hepatic steatosis. PLoS Biol 3:e101

10. Burgess SC, Leone TC, Wende AR et al (2006) Diminished hepatic gluconeogenesis via defects in tricarboxylic acid cycle flux in peroxisome proliferator-activated receptor gamma coactivator-1alpha (PGC-1alpha)-deficient mice. J Biol Chem 281:19000-19008

11. Handschin C, Lin J, Rhee J et al (2005) Nutritional regulation of hepatic heme biosynthesis and porphyria through PGC-1alpha. Cell 122:505-515

12. Lin J, Puigserver P, Donovan J, Tarr P, Spiegelman BM (2002) Peroxisome proliferator-activated receptor gamma coactivator 1 beta (PGC-1beta), a novel PGC-1-related transcription coactivator associated with host cell factor. J Biol Chem 277:1645-1648

13. Lin J, Tarr PT, Yang R et al (2003) PGC-1beta in the regulation of hepatic glucose and energy metabolism. J Biol Chem 278:3084330848 
14. O'Brien RM, Granner DK (1996) Regulation of gene expression by insulin. Physiol Rev 76:1109-1161

15. Herzog B, Hall RK, Wang XL, Waltner-Law M, Granner DK (2004) Peroxisome proliferator-activated receptor gamma coactivator-1alpha, as a transcription amplifier, is not essential for basal and hormone-induced phosphoenolpyruvate carboxykinase gene expression. Mol Endocrinol 18:807-819

16. Sladek FM (1993) Orphan receptor HNF-4 and liver-specific gene expression. Receptor 3:223-232

17. Gupta RK, Kaestner KH (2004) HNF-4alpha: from MODY to late-onset type 2 diabetes. Trends Mol Med 10:521-524

18. Chen WS, Manova K, Weinstein DC et al (1994) Disruption of the HNF-4 gene, expressed in visceral endoderm, leads to cell death in embryonic ectoderm and impaired gastrulation of mouse embryos. Genes Dev 8:2466-2477

19. Hayhurst GP, Lee YH, Lambert G, Ward JM, Gonzalez FJ (2001) Hepatocyte nuclear factor 4alpha (nuclear receptor 2A1) is essential for maintenance of hepatic gene expression and lipid homeostasis. Mol Cell Biol 21:1393-1403

20. Rhee J, Inoue Y, Yoon JC et al (2003) Regulation of hepatic fasting response by PPARgamma coactivator-1alpha (PGC-1): Requirement for hepatocyte nuclear factor 4alpha in gluconeogenesis. Proc Natl Acad Sci U S A 100:4012-4017

21. Boustead JN, Stadelmaier BT, Eeds AM et al (2003) Hepatocyte nuclear factor-4alpha (HNF-4alpha) mediates the stimulatory effect of peroxisome proliferator activated receptor gamma coactivator-1alpha (PGC-1alpha) on glucose-6-phosphatase catalytic subunit gene transcription in H4IIE cells. Biochem J 369:1722

22. Martin CC, Oeser JK, Svitek CA, Hunter SI, Hutton JC, O’Brien RM (2002) Identification and characterization of a human cDNA and gene encoding a ubiquitously expressed glucose-6-phosphatase catalytic subunit-related protein. J Mol Endocrinol 29:205222

23. Vanderbilt JN, Miesfeld R, Maler BA, Yamamoto KR (1987) Intracellular receptor concentration limits glucocorticoiddependent enhancer activity. Mol Endocrinol 1:68-74

24. Wang JC, Stafford JM, Granner DK (1998) SRC-1 and GRIP1 coactivate transcription with hepatocyte nuclear factor 4. J Biol Chem 273:30847-30850

25. Durham SK, Suwanichkul A, Scheimann AO et al (1999) FKHR binds the insulin response element in the insulin-like growth factor binding protein-1 promoter. Endocrinology 140:3140-3146

26. Puigserver P, Wu Z, Park CW, Graves R, Wright M, Spiegelman BM (1998) A cold-inducible coactivator of nuclear receptors linked to adaptive thermogenesis. Cell 92:829-839

27. Streeper RS, Svitek CA, Chapman S, Greenbaum LE, Taub R, O'Brien RM (1997) A multicomponent insulin response sequence mediates a strong repression of mouse glucose-6-phosphatase gene transcription by insulin. J Biol Chem 272:11698-11701

28. Streeper RS, Chapman SC, Ayala JE et al (1998) A phorbol esterinsensitive AP-1 motif mediates the stimulatory effect of insulin on rat malic enzyme gene transcription. Mol Endocrinol 12:1778-1791

29. Sambrook J, Fritsch EF, Maniatis EF (1989) Molecular cloning: a laboratory manual. Cold Spring Harbor Laboratory Press, Plainview

30. Ayala JE, Bracy DP, McGuinness OP, Wasserman DH (2006) Considerations in the design of hyperinsulinemic-euglycemic clamps in the conscious mouse. Diabetes 55:390-397

31. DeFronzo RA, Ferrannini E (1987) Regulation of hepatic glucose metabolism in humans. Diabetes Metab Rev 3:415-459
32. Pedersen KB, Zhang P, Doumen C et al (2007) The promoter for the gene encoding the catalytic subunit of rat glucose-6-phosphatase contains two distinct glucose-responsive regions. Am J Physiol Endocrinol Metab 292:E788-E801

33. Ayala JE, Streeper RS, Desgrosellier JS et al (1999) Conservation of an insulin response unit between mouse and human glucose-6phosphatase catalytic subunit gene promoters: transcription factor FKHR binds the insulin response sequence. Diabetes 48:18851889

34. Quandt K, Frech K, Karas H, Wingender E, Werner T (1995) MatInd and MatInspector: new fast and versatile tools for detection of consensus matches in nucleotide sequence data. Nucleic Acids Res 23:4878-4884

35. Osada S, Tsukamoto T, Takiguchi M, Mori M, Osumi T (1997) Identification of an extended half-site motif required for the function of peroxisome proliferator-activated receptor alpha. Genes Cells 2:315-327

36. Scott DK, Stromstedt PE, Wang JC, Granner DK (1998) Further characterization of the glucocorticoid response unit in the phosphoenolpyruvate carboxykinase gene. The role of the glucocorticoid receptor-binding sites. Mol Endocrinol 12:482-491

37. Onuma H, Vander Kooi BT, Boustead JN, Oeser JK, O'Brien RM (2006) Correlation between FOXO1a (FKHR) and FOXO3a (FKHRL1) binding and the inhibition of basal glucose-6-phosphatase catalytic subunit gene transcription by insulin. Mol Endocrinol 20:2831-2847

38. Vander Kooi BT, Streeper RS, Svitek CA, Oeser JK, Powell DR, O'Brien RM (2003) The three insulin response sequences in the glucose-6-phosphatase catalytic subunit gene promoter are functionally distinct. J Biol Chem 278:11782-11793

39. Schmoll D, Walker KS, Alessi DR et al (2000) Regulation of glucose-6-phosphatase gene expression by protein kinase Balpha and the forkhead transcription factor FKHR. Evidence for insulin response unit-dependent and -independent effects of insulin on promoter activity. J Biol Chem 275:36324-36333

40. Puigserver P, Rhee J, Donovan J et al (2003) Insulin-regulated hepatic gluconeogenesis through FOXO1-PGC-1alpha interaction. Nature 423:550-555

41. Schilling MM, Oeser JK, Boustead JN, Flemming BP, O'Brien RM (2006) Gluconeogenesis: re-evaluating the FOXO1-PGC1alpha connection. Nature 443:E10-E11

42. Barthel A, Schmoll D, Unterman TG (2005) FoxO proteins in insulin action and metabolism. Trends Endocrinol Metab 16:183189

43. Nasrin N, Ogg S, Cahill CM et al (2000) DAF-16 recruits the CREB-binding protein coactivator complex to the insulin-like growth factor binding protein 1 promoter in HepG2 cells. Proc Natl Acad Sci U S A 97:10412-10417

44. Puigserver P, Adelmant G, Wu Z et al (1999) Activation of PPARgamma coactivator-1 through transcription factor docking. Science 286:1368-1371

45. Matsumoto M, Pocai A, Rossetti L, Depinho RA, Accili D (2007) Impaired regulation of hepatic glucose production in mice lacking the forkhead transcription factor foxo1 in liver. Cell Metab 6:208216

46. Lei KJ, Shelly LL, Pan CJ, Sidbury JB, Chou JY (1993) Mutations in the glucose-6-phosphatase gene that cause glycogen storage disease type 1a. Science 262:580-583

47. Shelly LL, Lei KJ, Pan CJ et al (1993) Isolation of the gene for murine glucose-6-phosphatase, the enzyme deficient in glycogen storage disease type 1A. J Biol Chem 268:21482-21485 